\title{
Urinothorax: A rare complication of spontaneous bladder rupture
}

\author{
Cyrus Veryl Edelson*1, Scott Francis David Edelson², Jerome Craig Edelson ${ }^{3}$, Guy S. Dooley ${ }^{3}$ \\ ${ }^{1}$ Department of Medicine,Brooke Army Medical Center, San Antonio, United States \\ ${ }^{2}$ Department of Medicine, Medical University of South Carolina, Charleston, United States \\ ${ }^{3}$ Division of Gastroenterology and Hepatology, Brooke Army Medical Center, San Antonio, United States
}

Received: July 13, 2019

DOI: $10.5430 /$ crim.v6n4p16
Accepted: September 18, 2019 Online Published: September 29, 2019

URL: https://doi.org/10.5430/crim.v6n4p16

\begin{abstract}
Urinothorax (UT) is an uncommon cause of pleural effusion where urine collects in the pleural cavity that is usually caused by trauma or urinary obstruction. Spontaneous bladder rupture is another rare condition that is commonly caused by underlying bladder wall pathology and exacerbated by binge alcohol consumption, malignancy, autoimmune disorders, radiation, and obstructive or neurogenic pathology. We present a case of urinothorax caused by spontaneous bladder rupture in an adult patient successfully recognized and treated.
\end{abstract}

Key Words: Urinothorax, Spontaneous bladder rupture

\section{INTRODUCTION}

Urinothorax (UT) is an uncommon cause of pleural effusion that was first characterized in 1968 and has since then been only described in case reports. ${ }^{[1,2]}$ As such, the exact prevalence of this rare condition is unknown. The most common causes of UT are urinary obstruction, trauma, or iatrogenic as a result of GU manipulation or instrumentation. In rare cases, UT can also be caused by spontaneous bladder rupture (SRUB), an event that carries high morbidity and mortality if not promptly diagnosed and treated. Many cases of bladder rupture are often due to an inciting cause or underlying pathology however true idiopathic rupture is extremely rare. ${ }^{[3]}$ The anatomical location of the bladder within the pelvis makes it generally well contained and protected from most injuries. ${ }^{[3]}$ Currently, no published guidelines for management exist and treatment options range from invasive open surgical repair, to cystoscopy with endoscopic repair, and conservative management with placement of indwelling urinary catheters. ${ }^{[4]}$ Some cases of SRUB are diagnosed on the basis of elevated ascitic fluid creatinine concentrations; however, many patients do not show classic features of bladder rupture. We present a patient with unexplained ascites and a pleural effusion who was found to have UT due to SRUB.

\section{CASE PRESEntation}

A 46-year-old female presented to the emergency department for generalized abdominal pain complicated by new-onset dyspnea. She was taking multiple over the counter NSAIDs for ovarian cysts. She had a prior surgical history of a remote partial hysterectomy and two cesarean sections several years prior. She did not have a history of alcohol use, history of bladder manipulation or instrumentation, or have any recent abdominal or pelvic surgeries. A CT scan obtained in the ED revealed a very large right pleural effusion and resultant left midline shift with large volume intra-abdominal ascites with

\footnotetext{
*Correspondence: Cyrus Veryl Edelson; Email: Edelson.cyrus@gmail.com; Address: Department of Internal Medicine, Brooke Army Medical Center 3551 Roger Brooke Dr. Fort Sam Houston, TX 78234, United States.
} 
normal liver, bladder, spleen, pancreas, and adrenals (see Fig- in her ascites and pleural effusion.

ure 1). Initial labs revealed acute renal failure with a BUN (30 mg/dl), creatinine $(3.82 \mathrm{mg} / \mathrm{dl})$, and mild hyponatremia. Liver function enzymes were normal. Nephrology was consulted and a renal biopsy was obtained, which later showed findings consistent with acute tubular necrosis. Abdominal pain was unchanged and the patient's dyspnea progressively worsened. She was unable to receive diuretics due to her acute renal failure. As such, diagnostic and therapeutic thoracentesis was pursued, and 2 liters of straw-colored pleural fluid was removed with subjective improvement in dyspnea. Pleural fluid analysis was consistent with a transudative process (see Table 1). Because of diagnostic uncertainty, a diagnostic and therapeutic paracentesis was performed that revealed a serum ascites-albumin gradient of 3.2, inconsistent with portal hypertension. Perplexed by discrepancies between the pleural fluid and ascitic fluid, gastroenterology was consulted to evaluate for underlying liver disease.

An extensive workup consisting of acute and chronic viral hepatitis serologies, ferritin, and autoimmune workup including anti-mitochondrial antibody and anti-nuclear antibodies and anti-smooth muscle antibody were negative. Cytology and cultures of ascitic fluid were also negative for infection and malignancy. Given the extensive negative work up and in the context of ongoing renal failure and reaccumulation of her pleural effusion, a pleural fluid creatinine level was sent and returned elevated at $9.4 \mathrm{mg} / \mathrm{dl}$. Urology was consulted, performed cystoscopy, and the diagnosis of SRUB was made (see Figure 2). Her bladder rupture was managed cystoscopically with patching and foley catheter placement. Over the next few days, her renal function improved and she was discharged with a foley catheter.

At time of outpatient follow up, her foley catheter was removed and she was noted to have significant improvement

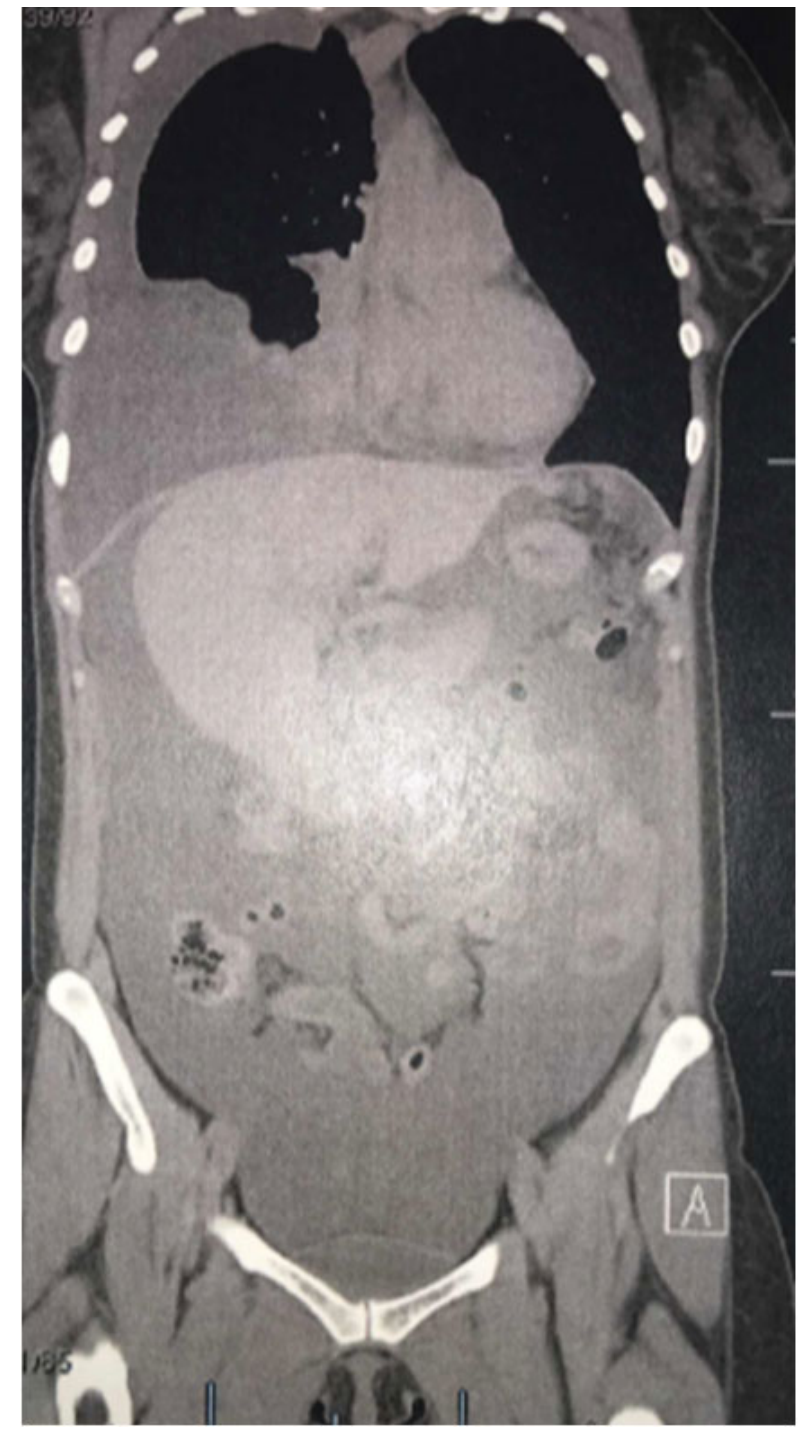

Figure 1. CT Chest/abdomen/pelvis

Table 1. Light's criteria

\begin{tabular}{lll}
\hline \multicolumn{1}{c}{ Test } & Sensitivity for Exudate & Specificity for Exudate \\
\hline Light's criteria (one or more of the following three) & $98 \%$ & $83 \%$ \\
Ratio of pleural-fluid protein level to serum protein level > 0.5 & $86 \%$ & $84 \%$ \\
Ratio of pleural-fluid $\mathrm{LDH}^{*}$ level to serum LDH level > 0.6 & $90 \%$ & $82 \%$ \\
Pleural-fluid LDH level > two thirds the upper limits of normal for serum & $82 \%$ & $89 \%$ \\
LDH level & & $92 \%$ \\
Pleural-fluid cholesterol level > 60 mg/dl (1.55 mmol/liter) & $54 \%$ & $80 \%$ \\
Pleural-fluid cholesterol level > 43 mg/dl (1.10 mmol/liter) & $75 \%$ & $81 \%$ \\
Ratio of pleural-fluid cholesterol level to serum cholesterol level >0.3 & $89 \%$ & $92 \%$ \\
Serum albumin level-pleural-fluid albumin level $\leqslant 1.2 \mathrm{~g} / \mathrm{dl}$ & $87 \%$ & \\
\hline
\end{tabular}

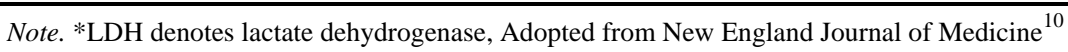




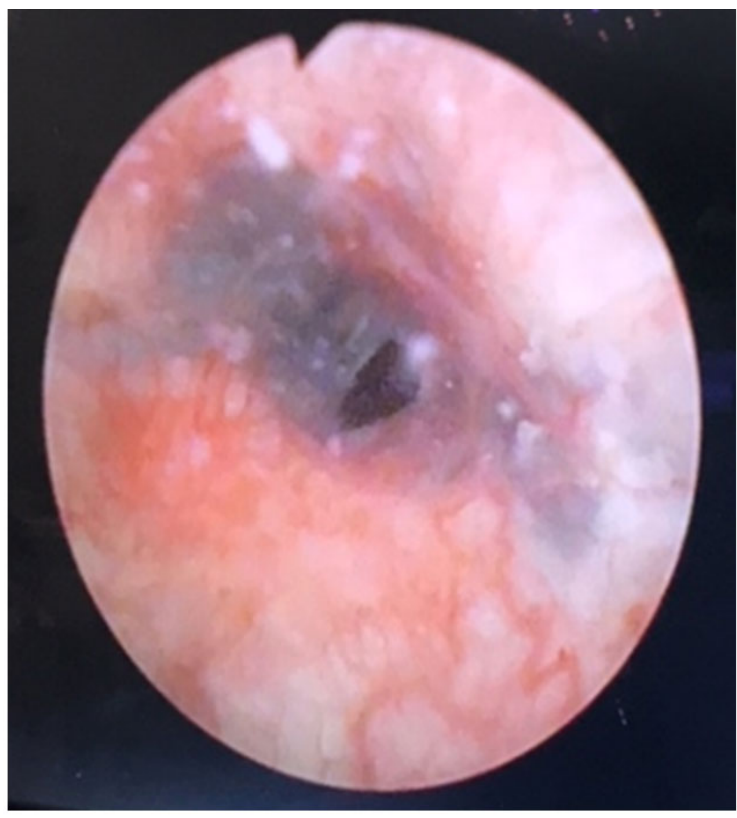

Figure 2. Cystoscopy

UT is an uncommon form of pleural effusion, which results from accumulation of urine within the pleural space. The most common etiologies are trauma or urinary obstruction, and a pleural fluid to serum $(\mathrm{PF} / \mathrm{S})$ creatinine ratio of greater than 1 supports the diagnosis. UT often develops rapidly over hours to days once a urinoma collects and classically presents with acute dyspnea $(92.8 \%)$, chest pain $(76.9 \%)$, abdominal pain $(91.4 \%)$, or reduced diuresis $(87 \%) .{ }^{[5,6]}$ Pleural fluid analysis of UT remains controversial as classically it was thought to be transudative, although a recent review reported exudative in $43.8 \%$ of cases. ${ }^{[6]}$ Unlike the prototypical patient who typically has a history of recent trauma or obstruction, our patient had SRUB. The majority of bladder ruptures occur as a result of trauma and typically manifest in several key presenting symptoms including gross hematuria and abdominal pain accompanied with peritoneal signs. ${ }^{[7]}$ In contrast to traumatic rupture, where the above signs are often present, spontaneous rupture presents a diagnostic dilemma as patients typically present with sub-clinical findings and often lack the known risk factors that would direct clinicians to this diagnosis. ${ }^{[7]}$ The incidence and prevalence of SRUB is not well known, and there are several case reports that attribute the etiology of rupture to underlying bladder wall pathology often exacerbated by binge alcohol consumption, malignancy, autoimmune disorders, radiation, and obstructive or neurogenic pathology. ${ }^{[3,7]}$ A thorough clinical history is key in identifying predisposing risk factors, although these factors may not be present in all patients as in our case. Our case was further complicated by the presence of acute renal failure, which could have masked the presence of hematuria, had urine been analyzed sooner in her clinical course. Treatment of UT is best accomplished in a multidisciplinary fashion with collaboration between urology, interventional radiology, and pulmonology. ${ }^{[8]}$ The first step in treatment involves drainage of UT for symptomatic relief followed by definitive repair of genitourinary tract pathology. Following definitive repair, symptoms associated with UT typically rapidly improve. ${ }^{[9]}$ As in our patient, she underwent cystoscopic repair followed by temporary urinary diversion with a foley catheter. Her symptoms rapidly improved with return of renal function to baseline and repeat imaging demonstrating resolution of UT and ascites. This case demonstrated an atypical cause of pleural effusion from an even more rare underlying cause. Prompt diagnosis enabled preservation of renal function and definitive repair of her ruptured bladder.

\section{CONFLicts OF InTEREST Disclosure}

The author declares no conflict of interest

\section{REFERENCES}

[1] Corriere JN, Jr., Miller WT, Murphy JJ. Hydronephrosis as a cause of pleural effusion. Radiology. 1968; 90: 79-84. PMid:4169467. https://doi.org/10.1148/90.1.79

[2] Kelley M, Spieler B, Rouse C, Karl B, Marshall R, Carbonella G. Urinothorax: A rare complication of percutaneous nephrostomy. Radiol Case Rep. 2019; 14: 729-733. PMid:30988865. https: //doi.org/10.1016/j.radcr.2019.03.022

[3] Parker H, Hoonpongsimanont W, Vaca F, Lotfipour S. Spontaneous bladder rupture in association with alcoholic binge: a case report and review of the literature. The Journal of emergency medicine. 2009;37:386-389. PMid:17976802. https ://doi.org/10.1016/ j.jemermed.2007.03.049

[4] Al-Qassim Z, Mohammed A, England R, Khan Z. Idiopathic spontaneous rupture of the urinary bladder (SRUB). A case report and review of literature. Central European journal of urology. 2012;65:235237. PMid:24578972. https://doi.org/10.5173/ceju. 2012. $04 . \operatorname{art} 14$
[5] Garcia-Pachon E, Romero S. Urinothorax: a new approach. Curr Opin Pulm Med. 2006;12:259-263. PMid:16825877. https://doi . org/10.1097/01.mcp.0000230628.65515.86

[6] Toubes ME, Lama A, Ferreiro L, et al. Urinothorax: a systematic review. J Thorac Dis. 2017;9:1209-1218.PMid:28616270. https : //doi.org/10.21037/jtd.2017.04.22

[7] Loganathan A, Wood J, Pridgeon S. Idiopathic spontaneous rupture of the urinary bladder: An unusual presentation of intraperitoneal bladder rupture managed conservatively. Urology case reports. 2019; 24: 100873. PMid:31211082. https://doi.org/10.1016/j.eu cr. 2019.100873

[8] Austin A, Jogani SN, Brasher PB, Argula RG, Huggins JT, Chopra A. The Urinothorax: A Comprehensive Review With Case Series. Am J Med Sci. 2017; 354: 44-53. PMid:28755732. https: //doi.org/10.1016/j.amjms.2017.03.034

[9] Casallas A, Castaneda-Cardona C, Rosselli D. Urinothorax: Case report and systematic review of the literature. Urol Ann. 2016; 8 : 91-94. PMid:26834411. https://doi.org/10.4103/0974-779 6.164851 